\title{
Heavy Ion Interactions from Coulomb Barrier to Few GeV/n: Boltzmann Master Equation Theory and FLUKA Code Performances
}

\author{
G. Battistoni, M. Cavinato, F. Cerutti, A. Clivio, E. Fabrici, \\ E. Gadioli, E. Gadioli Erba, M. V. Garzelli, A. Mairani \\ Milan University and INFN, Italy \\ A. Empl, L. S. Pinsky, \\ Houston University, Texas, USA \\ F. Ballarini, A. Ottolenghi, \\ Pavia University and INFN, Italy \\ A. Fassò, \\ SLAC, Stanford, USA \\ A. Ferrari, \\ CERN, Switzerland \\ J. Ranft, \\ Siegen University, Germany \\ and P. R. Sala \\ ETH, Zurich, Switzerland
}

Received on 8 October, 2003

Results which have been recently obtained with the Boltzmann master equation and the FLUKA code in the analysis of heavy ion interactions at relative energies ranging from Coulomb barrier up to a few $\mathrm{GeV} / \mathrm{n}$ are discussed.

\section{Introduction}

The importance of an accurate prediction of all what happens when a nuclear interaction takes place, i.e. of the cross sections of the reactions which may occur and of the spectra of particles, $\gamma$ rays and heavy residues which may be produced, cannot be underestimated from the point of view of both basic knowledge and the possible use of nuclear physics in interdisciplinary fields and applications useful to mankind such as medical diagnostics and therapy, dosimetry, environmental control, space physics, and industrial research. Satisfactory results were first obtained for nucleon induced reactions by MonteCarlo calculations and the subsequent pre-equilibrium theories [1] which provided the unification of seemingly different nuclear reaction theories. The extension of such results to heavy ion interactions is one of the goals of our collaboration, exploring different approaches. At incident energies up to about $50 \mathrm{MeV} / \mathrm{n}$ calculations considering both the mean field interaction between the two ions and the subsequent de-excitation via nucleonnucleon collisions of the highly excited composite nucleus which may be created, are performed with the Boltzmann Master Equation (BME) theory [2,3] and reproduce reliably a large set of data. At considerably higher energies various experiments are analysed using FLUKA (a transport and interaction MonteCarlo code capable of handling reactions induced by hadronic and electromagnetic particles ranging from thermal neutrons up to cosmic rays with several $\mathrm{TeV}$ incident energy $[4,5,6])$ coupled with a modified version of a Relativistic Quantum Molecular Dynamics (RQMD) code developed in Frankfurt [7, 8]. In this paper we show some recent results obtained for both energy ranges.

\section{BME calculations}

The BME theory, as formulated by our research group, is explained in several papers [2,3,9-11], so in this Section we limit ourselves to remind its main assumptions. It describes the thermalization of an excited nucleus by evaluating the variation with time of the distribution of the momenta of its nucleons as a result of their mutual interactions and their emission into the continuum as separate entities or as part of a cluster. The nucleon momentum space is divided into bins $i$ of volume $\Delta V=2 \pi m \Delta \epsilon \cdot \Delta p_{z}$ (where $m$ and $\epsilon$ are the nucleon mass and energy and $p_{z}$ is the component of nucleon momentum along the beam axis) and the time evolution of the occupation probability $n(\epsilon, \theta, t)$ of the states of each bin is calculated. To this aim a set of coupled differential equations, giving the variation of $n(\epsilon, \theta, t)$ in the time interval between $t$ and $t+d t$ as a function of the occupation probabilities at time $t$ and the decay rates for nucleonnucleon scatterings and emissions into the continuum, has to be integrated. 

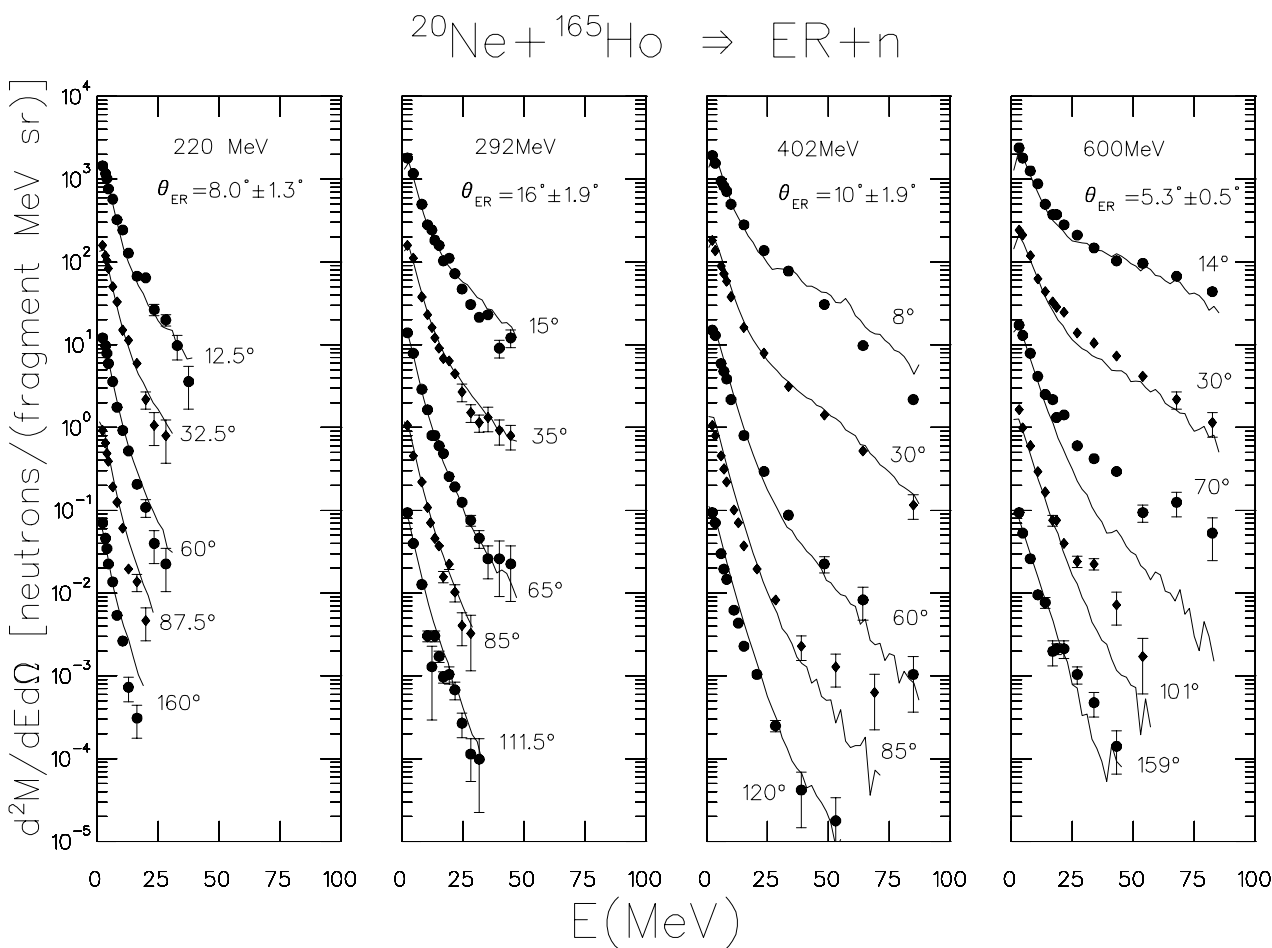

Figure 1. Spectra of neutrons in coincidence with a heavy residue emitted at a forward angle in the interaction of ${ }^{20} \mathrm{Ne}$ with ${ }^{165} \mathrm{Ho}$ at incident energies varying from 11 to $30 \mathrm{MeV} / \mathrm{n}$. The symbols give the experimental values [12, 13], the continuous lines the result of our calculation. Starting from bottom the spectra are progressively scaled up by a factor ten.

The information so obtained is inclusive, as averaged over many different reaction paths. In other words, the theory provides the mean multiplicities of emitted particles and does not allow one to analyse exclusive processes or evaluate other measurable quantities like, for instance, the cross sections for residue formation. In order to eliminate such limitations, the spectra predicted by BME's may be used as input for a MonteCarlo calculation assuming that the probability of emitting a particle $i$ with energy between $\epsilon$ and $\epsilon+d \epsilon$ at a polar angle between $\theta$ and $\theta+d \theta$ in the time interval $(t, t+d t)$ be equal to its expected differential multiplicity $d^{3} M_{i}(\epsilon, \theta, t)[3,11]$. The probability of any possible sequence of events may be evaluated as a joint probability using these elementary probabilities.

The effect of the mean field interaction, which dominates when the two nuclei are approaching each other, can be appreciated in Fig. 1 where the spectra of neutrons emitted in the complete fusion of ${ }^{20} \mathrm{Ne}$ with ${ }^{165} \mathrm{Ho}$ at bombarding energies varying from 11 to $30 \mathrm{MeV} / \mathrm{n}$ are shown $[12,13]$. At the lowest incident energy the emission of pre-equilibrium neutrons is hindered because it occurs while the two interacting nuclei, which are slowed down by their Coulomb repulsion, still form a di-nuclear system and a large fraction of their energy is in form of collective deformation energy [10]. On the other hand, at the highest incident energy the pre-equilibrium contribution is enhanced since the projectile's and target's nucleons increase their energy falling into a deeper potential well [9].

In its most recent version the BME theory could satis- factorily account for the emission of intermediate mass fragments (IMF) produced by nucleon coalescence. An example of such results is given in Fig. 2, showing the spectra of boron fragments produced in the interaction of ${ }^{16} \mathrm{O}$ with ${ }^{93} \mathrm{Nb}$ at $25 \mathrm{MeV} / \mathrm{n}$. The BME theory predicts that the IMF's emitted during the pre-equilibrium de-excitation display a quasi - evaporative spectrum peaked at low energy, becoming the dominant contribution to the measured spectrum at sufficiently large emission angles. As shown in the figure, as for reactions induced by light ions, break-up processes also contribute to a considerable extent to the reaction cross section, accounting for the high energy peak at forward angles.

\section{RQMD calculations}

At considerably higher energies than those considered in previous calculations, the QMD models represent a suitable way to treat nucleus-nucleus collisions thanks to their dynamical modelling of the nuclear field among nucleons in the course of the reaction. The RQMD-2.4 code $[7,8]$ was successfully applied to particle production in relativistic heavy ion interactions over a wide energy range. A modified version of this code has been recently interfaced with FLUKA [15] and used to analyse experiments providing measurements of double differential neutron yield and fragment cross sections at several hundred of $\mathrm{MeV} / \mathrm{n}$. It is outside our present scope to review the basic assumptions of 

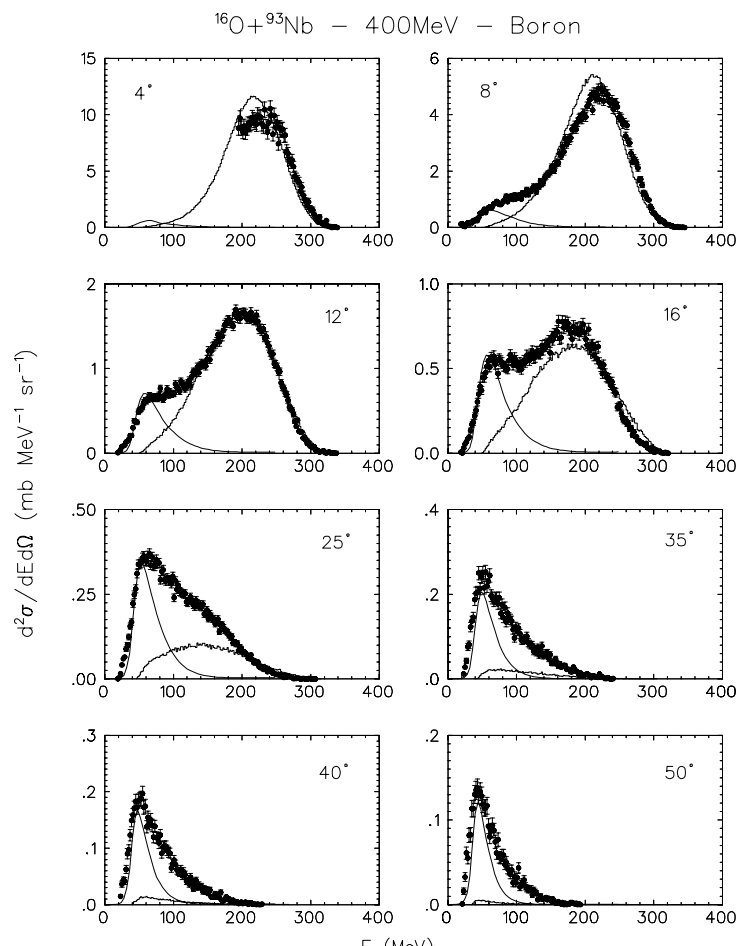

Figure 2. Spectra of boron fragments emitted in the interaction of ${ }^{16} \mathrm{O}$ with ${ }^{93} \mathrm{Nb}$ at $25 \mathrm{MeV} / \mathrm{n}$ [14]. Symbols represent the experimental data, full lines the coalescence contribution, and histograms the contribution given by the binary fragmentation of the projectile.

the well known QMD approach and here we limit ourselves to mention a few innovations we have implemented in the original code of the Frankfurt group.

RQMD-2.4 was not identifying the residual nuclei in the final state. Thus their de-excitation by pre-equilibrium emissions, fission, evaporation, or fragmentation could not be evaluated. This is a quite severe limitation: in fact, because of huge kinematical effects, in some instances ejectiles having low energy in the reference frame of the decaying projectile-like nucleus may contribute to the highest energy side of observed spectra at forward angles. We have tentatively identified projectile- and target-like residues by gathering spectator nucleons and calculated their excitation energy from the actual depth of the holes left by the hit nucleons. This simple development allowed us to estimate their final de-excitation by the FLUKA evaporation module and to obtain, e.g., the reasonable reproduction, shown in Fig. 3, of double differential neutron spectra measured by $\mathrm{T}$. Kurosawa $e t$ al. delivering a beam of $\mathrm{Fe}$ ions at $400 \mathrm{MeV} / \mathrm{n}$ on a thick Al target [16]. Moreover, energy non-conservation issues, which affected the original code at bombarding energies of our interest, have been solved taking into account the experimental binding energies of nuclei, as is the case for all other models used in FLUKA. This way, a meaningful evaluation of observables which critically

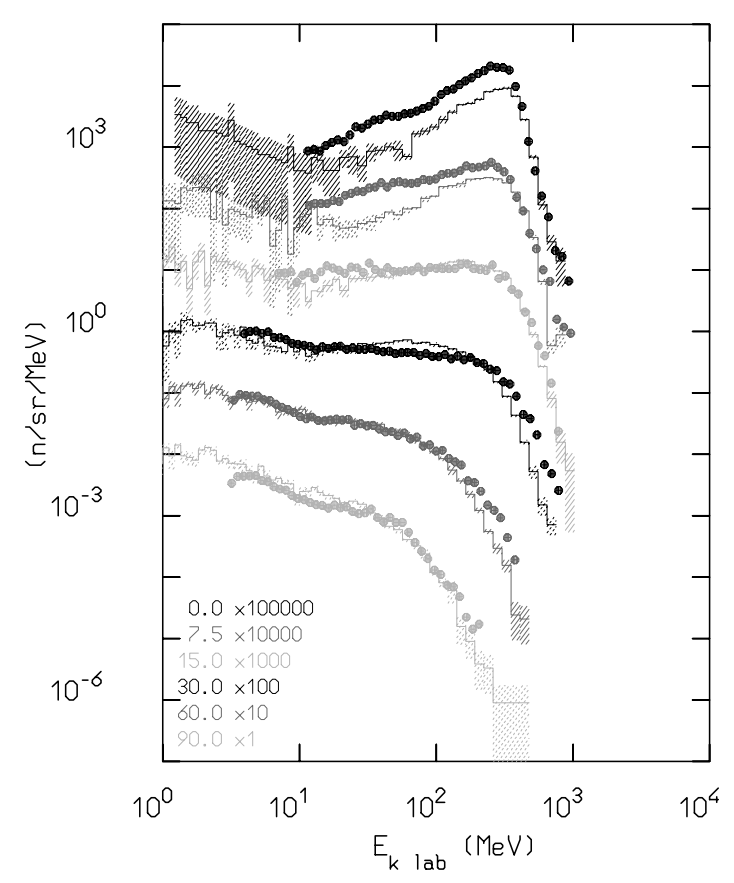

Figure 3. Double differential neutron yield by $400 \mathrm{MeV} / \mathrm{n}$ Fe ions on a thick $\mathrm{Al}$ target. Dots represent the experimental data from [16], histograms the FLUKA outcome.

depend on the exact bookkeeping of the excitation energy, like e.g. fragment mass distributions, became possible. Fig. 4 shows the satisfactory agreement between theoretical calculation and experimental data as for the isotopic distributions of fragmentation products in the reaction ${ }^{238} \mathrm{U}+$ ${ }^{208} \mathrm{~Pb}$ at $750 \mathrm{MeV} / \mathrm{n}$.

\section{Concluding remarks}

The results that we have shown suggest that it is really possible to perform accurate and comprehensive calculations for the processes that occur when two heavy ions interact at energies ranging from their Coulomb barrier up to several hundred of $\mathrm{MeV} / \mathrm{n}$. Of course the outlined models may be improved in many ways, however just now they provide an extremely useful and powerful means of investigation in different fields.

\section{Acknowledgments}

This work was partially supported under NASA Grants NAG8-1658 and 01-OBPR-05, ASI contract 1/R/320/02, DoE contract DE-AC03-76SF00515 and EC contract FIGHCT1999-00005. 


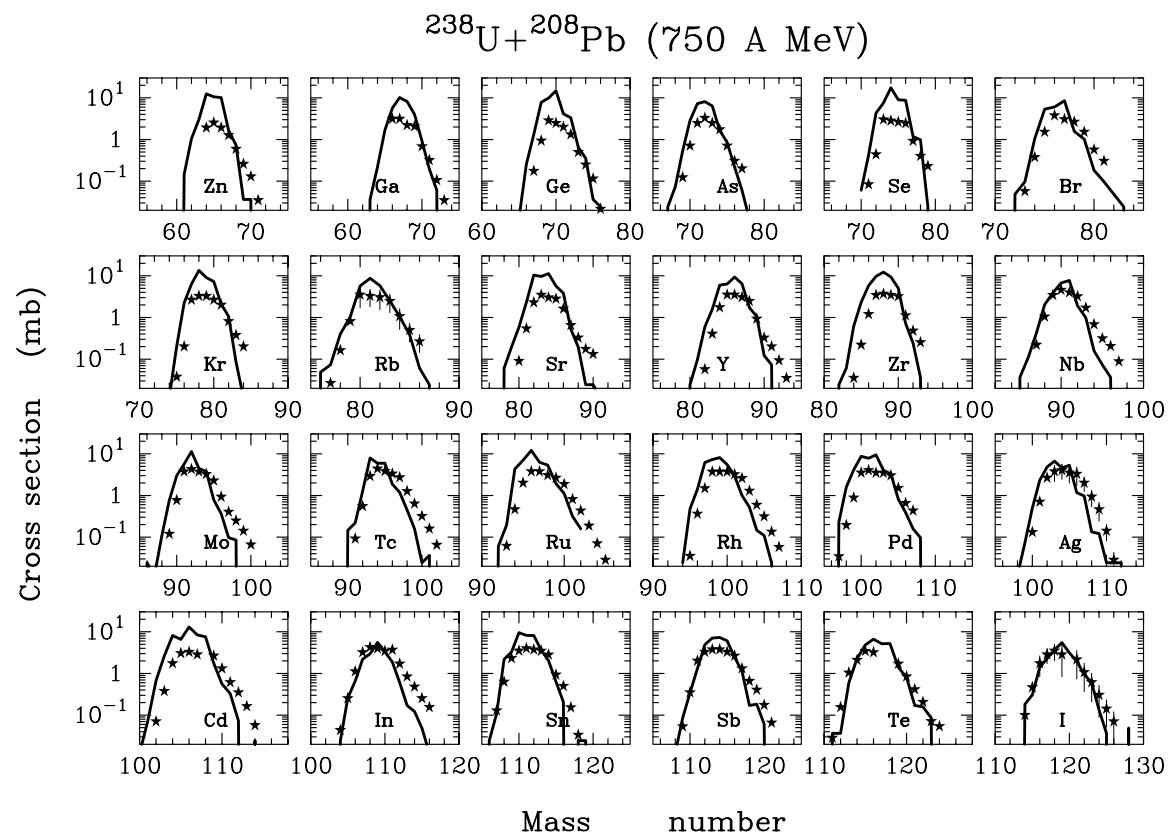

Figure 4. Isotopic distributions of fragmentation products for $750 \mathrm{MeV} / \mathrm{n} \mathrm{U}$ ions on $\mathrm{Pb}$. Data (stars) from [17]. The fission products have been excluded in the experimental analysis and so in our calculation (full lines) too.

\section{References}

[1] E. Gadioli and P.E. Hodgson, Pre-Equilibrium Nuclear Reactions, Oxford Science Publications, Clarendon Press Oxford, 1992.

[2] M. Cavinato et al., Nucl. Phys. A643, 15 (1998).

[3] M. Cavinato et al., Nucl. Phys. A679, 753 (2001).

[4] A. Fassò, A. Ferrari, J. Ranft, and P.R. Sala, Proceedings of SARE-3, KEK-Tsukuba, H. Hirayama ed., May 7-9 1997, KEK report Proceedings 97-5, 32 (1997).

[5] A. Fassò, A. Ferrari, J. Ranft, and P.R. Sala, Proceedings of the MonteCarlo 2000 Conference, Lisbon, October 23-26 2000, A. Kling, F. Barão, M. Nakagawa, L. Távora, P. Vaz eds., Springer-Verlag Berlin, 955 (2001).

[6] A. Fassò, A. Ferrari and P.R. Sala, Proceedings of the MonteCarlo 2000 Conference [5], 159 (2001).
[7] H. Sorge, H. Stöcker and W. Greiner, Nucl. Phys. A498, 567c (1989); Ann. Phys. 192, 266 (1989).

[8] H. Sorge, Phys. Rev. C52, 3291 (1995).

[9] C. Brusati et al., Z. Phys. A353, 57 (1995).

[10] M. Cavinato et al., Phys. Rev. C52, 2577 (1995).

[11] M. Cavinato et al., Phys. Lett. B382, 1 (1996).

[12] E. Holub et al., Phys. Rev. C28, 252 (1983).

[13] D. Hilscher et al., Phys. Rev. C36, 208 (1987).

[14] E. Gadioli et al., Eur. Phys. J. A17, 195 (2001).

[15] V. Andersen et al., Adv. Space Res., in press.

[16] T. Kurosawa et al., Phys. Rev. C62, 044615-1 (2000).

[17] J. Benlliure et al., Eur. Phys. J. A2, 193 (1998). 